\title{
The Void Galaxy Survey: Morphology and Star Formation Properties of Void Galaxies
}

\author{
Burcu Beygu ${ }^{1}$, Kathryn Kreckel ${ }^{2}$,Thijs van der Hulst ${ }^{1}$, \\ Reynier Peletier ${ }^{1}$, Tom Jarrett ${ }^{3}$, Rien van de Weygaert ${ }^{1}$, \\ Jacqueline H. van Gorkom ${ }^{4}$ and Miguel Aragón-Calvo ${ }^{5}$ \\ ${ }^{1}$ Kapteyn Astron. Inst., Univ. Groningen, PO Box 800, 9700 AV Groningen, the Netherlands \\ email: beygu@astro.rug.nl \\ ${ }^{2}$ MPIA, Königstuhl 17, 69117 Heidelberg, Germany \\ ${ }^{3}$ Dept. Astron., Univ. Cape Town, Private Bag X3, Rondebosch 7701, South Africa \\ ${ }^{4}$ Columbia University, MC 5246, 550 W120th St., New York, NY 10027, USA \\ ${ }^{5}$ University of California, Riverside, CA 92521, USA
}

\begin{abstract}
We present the structural and star formation properties of 59 void galaxies as part of the Void Galaxy Survey (VGS). Our aim is to study in detail the physical properties of these void galaxies and study the effect of the void environment on galaxy properties. We use Spitzer $3.6 \mu \mathrm{m}$ and B-band imaging to study the morphology and color of the VGS galaxies. For their star formation properties, we use $\mathrm{H} \alpha$ and GALEX near-UV imaging. We compare our results to a range of galaxies of different morphologies in higher density environments. We find that the VGS galaxies are in general disk dominated and star forming galaxies. Their star formation rates are, however, often less than $1 \mathrm{M}_{\odot} \mathrm{yr}^{-1}$. There are two early-type galaxies in our sample as well. In $\mathrm{r}_{\mathrm{e}}$ versus $\mathrm{M}_{\mathrm{B}}$ parameter space, VGS galaxies occupy the same space as dwarf irregulars and spirals.
\end{abstract}

\section{Voids and void galaxies}

Voids are a prominent aspect of the Cosmic Web (see van de Weygaert \& Platen (2011) for a review). Surrounded by elongated filaments, sheetlike walls and dense compact clusters, they have formed out of primordial underdensities via an intricate hierarchical process of evolution (Sheth \& van de Weygaert (2004), Aragón-Calvo et al. (2010), Aragón-Calvo \& Szalay (2013), Rieder et al. (2013)). Their diluted substructure and population of galaxies remain as fossils of the earlier phases of this void hierarchy.

Within this context, the pristine environment of voids represents an ideal setting for the study of environmental influences on galaxy formation and evolution. Largely unaffected by the complexities and processes that modify galaxies in high-density environments, the characteristics of void galaxies are expected to provide information on the role of environment in galaxy evolution.

A few aspects stand out immediately. The void galaxies are in general gas rich and display a substantial star formation activity. They also have rather low stellar masses, which relates to the finding by theoretical studies that the mass function of galaxies and halos in voids has shifteed considerably towards lower masses (see e.g. Goldberg \& Vogeley (2004), Aragón-Calvo (2007), Cautun et al. (2014)). What still remains to be understood is the remarkable low abundance of dwarf galaxies in void interiors (Peebles (2001)). Also, we may wonder in how far the gas accretion and outflow history of void 
Spiral

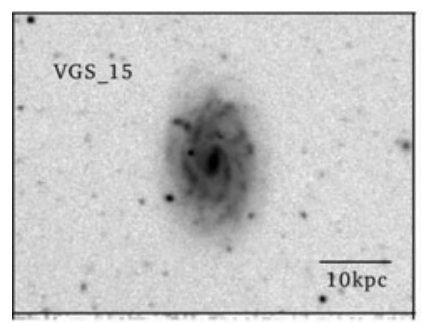

Edge-on

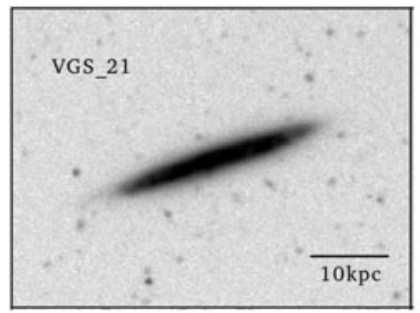

Early-type

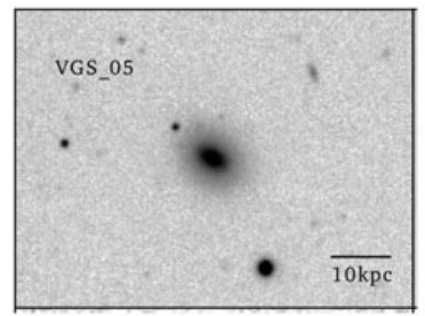

Irregular

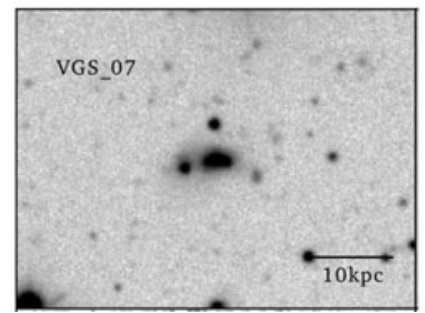

Compact

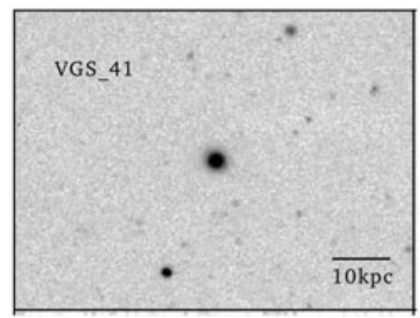

AGN

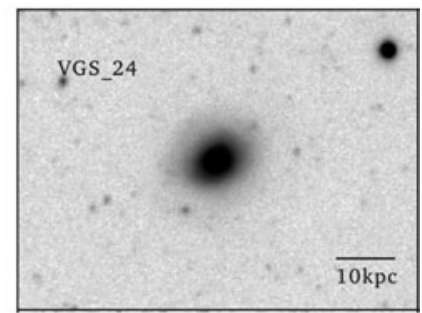

Figure 1. VGS galaxy morphologies. The images are B-band images of 6 VGS galaxies. In each image, the black bar represents a physical scale of $10 \mathrm{kpc}$.

galaxies has resulted in systematically different objects (e.g. Keres et al. (2005), Hoeft \& Gottlöber (2010)).

In an attempt to obtain more insight into the properties of void galaxies, in this study we explore the morphology, structural characteristics and star formation properties of void galaxies in the Void Galaxy Survey (VGS).

\section{The Void Galaxy Survey}

In order to fully study the effect of void environment on galaxy evolution and formation, one needs an unbiased void galaxy sample found in a well defined void environment. The Void Galaxy Survey (VGS) (Kreckel et al. (2011), van de Weygaert et al. (2011), Kreckel et al. (2012), this volume) provides such a sample. This sample has been selected from the Sloan Digital Sky Survey Data Release 7 (SDSS DR7), using geometric and topological techniques for delineating voids in the galaxy distribution and identifying galaxies populating the central interior of these voids (Schaap \& van de Weygaert (2000), Platen et al. (2007), Aragón-Calvo et al. (2010), Kreckel et al. (2011)). The typical size of voids in our sample is on the order of 5 to $10 h^{-1} \mathrm{Mpc}$ in radius.

The resulting sample of void galaxies is unbiased and largely independent of intrinsic galaxy properties (except for the the spectroscopic flux limit of 17.7 mag in the r-filter of the SDSS). The VGS galaxies have redshifts in the range $0.02<\mathrm{z}<0.03$. They have an absolute magnitude in the range of $-20.4<\mathrm{M}_{\mathrm{r}}<-16.1$, colors in between $0.06<\mathrm{g}-\mathrm{r}<0.087$ and a stellar mass $\mathrm{M}_{*}<3 \times 10^{10} \mathrm{M}_{\odot}$. The Void Galaxy Survey aims to probe the color, morphology, star formation and gas content of the void galaxies. For this we observed 59 VGS galaxies in the $21 \mathrm{~cm} \mathrm{Hi} \mathrm{line,} \mathrm{in} \mathrm{H} \alpha$ and in the optical B-band. In addition, we acquired GALEX near-UV data, as well as Spitzer $3.6 \mu \mathrm{m}$ and WISE $22 \mu \mathrm{m}$ imaging. For some VGS galaxies we obtained $\mathrm{CO}(1-0)$ observations, which will form the starting point for a study of the relation between their star formation activity and their molecular and atomic gas content. 


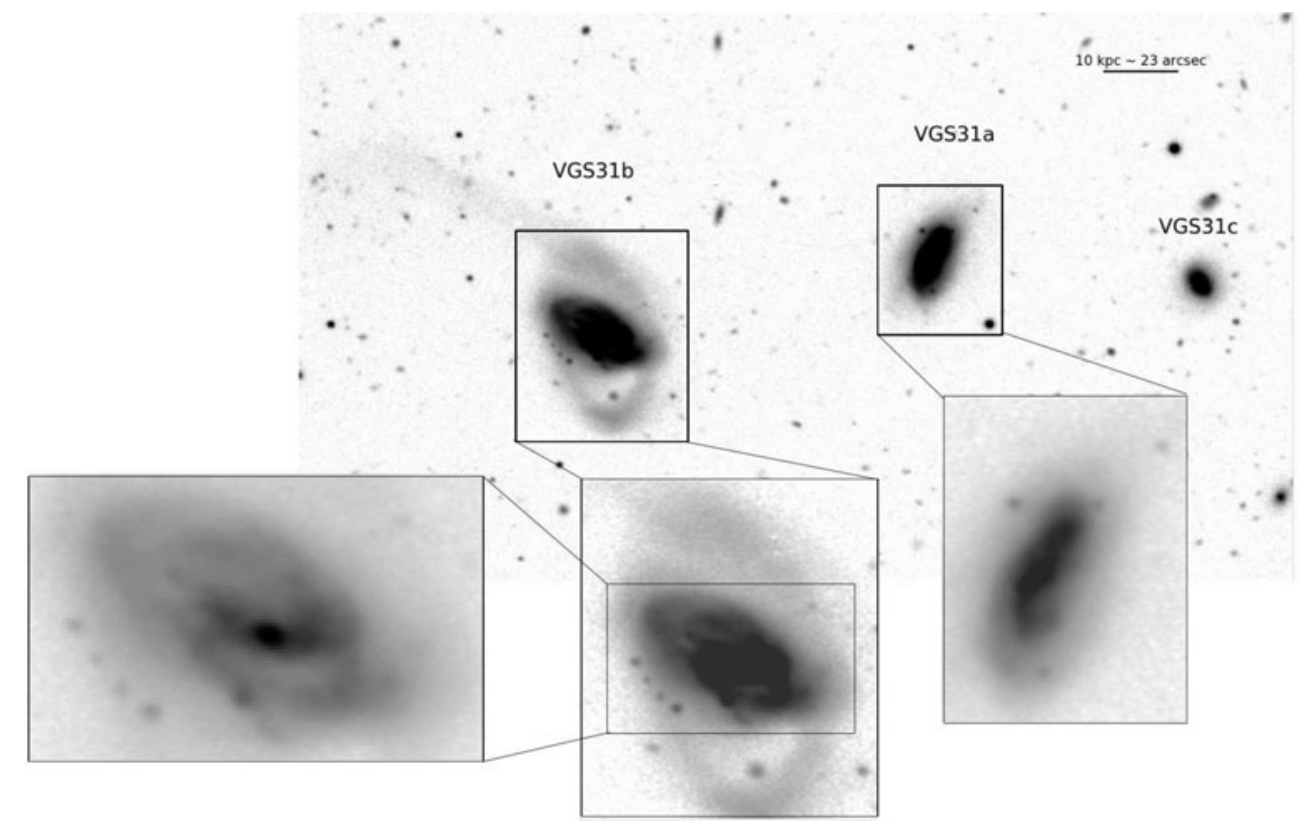

Figure 2. VGS_31. R-band negative image of this configuration of three void galaxies aligned along a tenuous filament inside the void. From left to right: VGS_31b: The most remarkable member of the system, a Markarian galaxy, has a tail and a ring. Close up images show the inner structures such as the bar. VGS_31a: A disk galaxy with a bar structure. VGS_31c: Smallest member of the system is optically undisturbed. The black bar on the top-right corner represents $10 \mathrm{kpc}\left(\sim 23^{\prime \prime}\right)$ (Beygu et al. $(2013)$ ).

So far we have completed the study of the Hi properties of 55 VGS galaxies (see Kreckel et al. (2012) for details ).

\section{Morphology \& Structural Parameters}

The morphological classification of galaxies is rather complex and an accurate classification requires a more elaborate analysis than we are able to provide here. Therefore, instead of carrying out an absolute morphological classification, we seek to classify the morphology of the VGS galaxies in a general way, by eye. We find that the VGS galaxy sample mainly consists of disk galaxies with an occasional bar and spiral structure and sometimes small compact objects, irregulars and two early-types (one is an AGN). In figure 1 we display examples of the different morphological types of galaxies that we find in the VGS sample. Also, we find various peculiar galaxies, such as the dynamically distorted Markarian galaxy VGS_31b in the filamentary VGS31 constellation (figure 2, see Beygu et al. (2013)).

\subsection{Structural parameters}

The structural analysis of the VGS galaxies involves the fitting of Sérsic profiles to the light distributions and the determination of the characteristic size $\left(\mathrm{r}_{\mathrm{e}}\right)$ and surface brightness $\left(\mu_{\mathrm{e}}\right)$ of the galaxies, along with their total luminosity. The concentration of (stellar) light is quantified by the Sérsic index $n$. We have compared the determined structural parameter values for the VGS galaxies with the values of the same parameters for a wide range of different galaxies. Amongst these are giant early-type galaxies, dEs and late-type disk galaxies. Figure 3a shows a sketch of where different types of galaxies are 


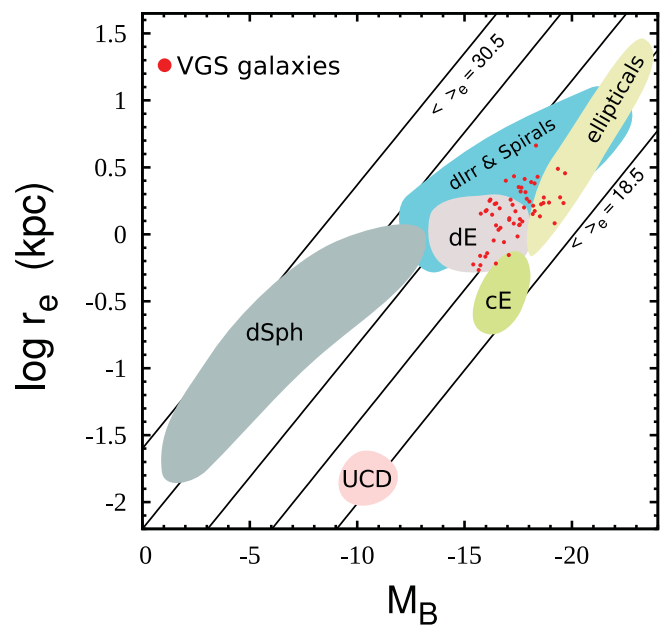

(a)

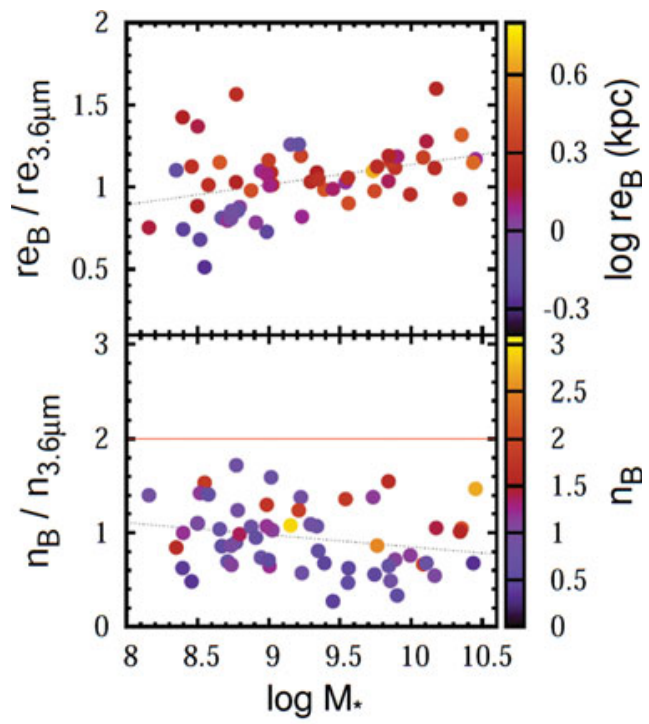

(b)

Figure 3. VGS galaxy structural parameters. (a): A sketch showing regions occupied by different types of galaxies and the VGS galaxies in the parameter space of the half-light radii $r_{e}$ and B-band absolute magnitude $\mathrm{M}_{\mathrm{B}}$ adopted from Mo et al. (2010). VGS galaxies are shown as red dots. (b): The ratios of the $\mathrm{r}_{\mathrm{e}}$ (top) and Sérsic indices (bottom) of the B-band and $3.6 \mu \mathrm{m}$ as a function of stellar mass, $r_{e(B)}$ and Sérsic index $n_{B}$.

located in terms of their half-light radii and B-band absolute magnitudes. VGS galaxies (red dots), dIrr and spirals occupy the same space in $r_{e}$ versus $M_{B}$.

Figure $3 b$ shows the $r_{e, B} / r_{e, 3.6}$ (top panel) and the $n_{B} / n_{3.6}$ ratios (lower panel) as a function of stellar mass, $r_{e, B}$ and Sérsic index $n_{B}$. The majority of the VGS galaxies have Sérsic indices $n<2$ in both bands. This confirms that they are disk dominated.

In figure $3 \mathrm{~b}$, we see that $\mathrm{r}_{\mathrm{e}, \mathrm{B}} / \mathrm{r}_{\mathrm{e}, 3.6}$ increases as a function of increasing stellar mass and as as well as a function of increasing $\mathrm{r}_{\mathrm{e}, \mathrm{B}}$. In other words, the smaller galaxies have a more concentrated light distribution at $3.6 \mu \mathrm{m}$ than in B. One explanation may be that in smaller galaxies the star formation activity is more concentrated towards the center than in the larger objects. Part of the effect is also caused by extinction. Larger galaxies generally contain more dust in their central regions. Dust affects the light in the B-band more than at $3.6 \mu \mathrm{m}$. This translates into a larger $\left(\mathrm{r}_{\mathrm{e}}\right)_{\mathrm{B}}$ than $\left(\mathrm{r}_{\mathrm{e}}\right)_{3.6}$.

\section{Star formation properties}

From $\mathrm{H} \alpha$ and near-UV imaging we may conclude that VGS galaxies are galaxies with a substantial star forming activity. Nonetheless, most of them appear to have star formation rates less than $1 \mathrm{M}_{\odot} \mathrm{yr}^{-1}$. The one exception with a considerably elevated star formation rate is the VGS_31 (Beygu (2014)) system.

We have compared the specific star formation $\left(\mathrm{SFR}_{\alpha} / \mathrm{M}_{*}\right)$ and star formation efficiencies - i.e. $\mathrm{SFR}_{\alpha} / \mathrm{M}_{\mathrm{HI}}$ - of the VGS galaxies to those of galaxies in average density regions (see Beygu (2014) for details). The latter belong to a sample of galaxies that consists of a combination of galaxies defined by three studies (Gavazzi et al. (2012), Sánchez-Gallego et al. (2012) and Karachentsev et al. (2013)). These cover the same stellar mass range, as well as other properties, as the VGS galaxies. 

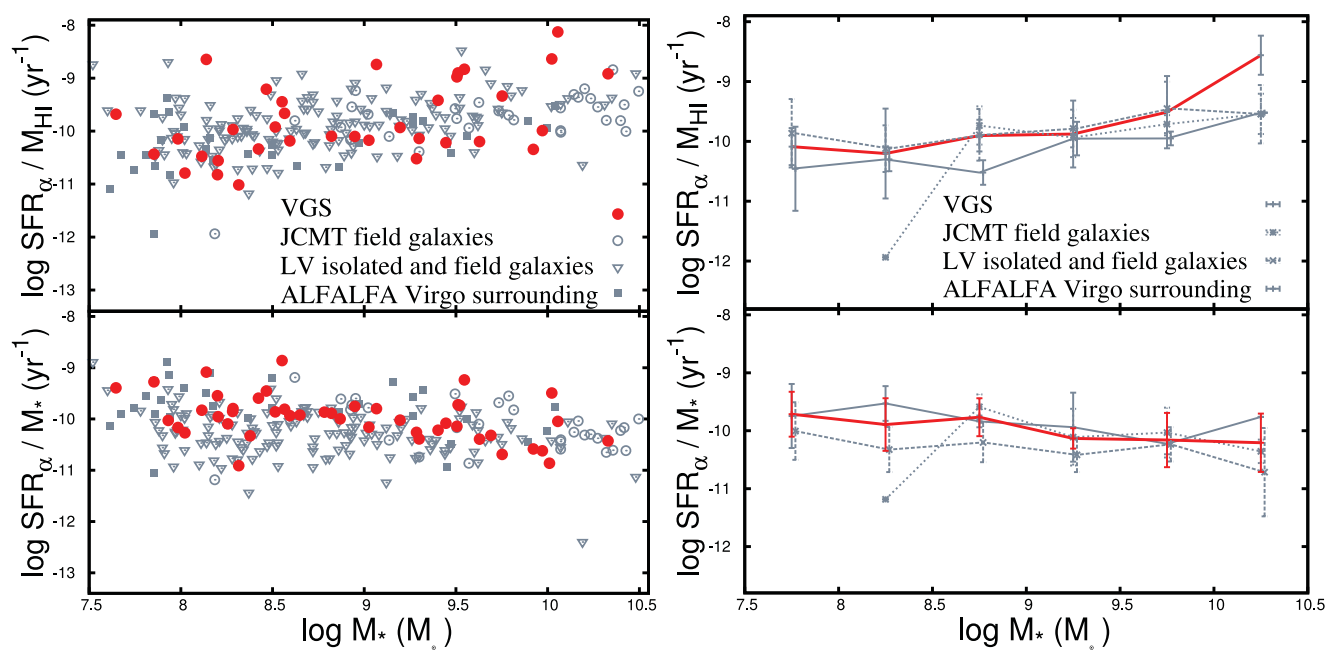

Figure 4. Star formation properties of VGS galaxies. Top left: $\mathrm{SFR}_{\alpha} / \mathrm{M}_{\mathrm{HI}}$ as a function of the stellar masss $\mathrm{M}_{*}$. VGS galaxies are indicated by red dots, the comparison sample galaxies are indicated by faint symbols. Bottom left: $\mathrm{SFR}_{\alpha} / \mathrm{M}_{*}$ as a function of stellar mass $\mathrm{M}_{*}$ for the sample sample of galaxies. Right panels: the average of the specific star formation parameters plotted in the corresponding lefthand panels. Note that there does not appear to be a significant difference between the VGS and the comparison sample galaxies.

The left panel of figure 4 reveals a similar dependence of star formation efficiency and specific star formation rate on stellar mass for the VGS galaxies as for the galaxies from the control samples. As a function of stellar mass, the VGS and control sample galaxies show the same weak trends. In other words, we do not seem to detect a significant difference between the VGS galaxies and the control sample galaxies.

\section{Conclusion}

The voids in our VGS sample do not appear to be populated by a type of galaxy specific for void environments. Voids mainly contain late-type galaxies of different morphologies. Only two VGS galaxies have an early-type morphology. While the void environment expresses itself in the low stellar mass and size of the galaxies (see e.g. Aragón-Calvo (2007), Cautun et al. (2014)), there is not evidence for star formation activity that deviates significantly from their peers with a similar mass in the higher denstiy filamentary and cluster-like environments of the cosmic web. It forms an indication for star formation to be a mainly self regulated process, not strongly influenced by the large scale environment. To understand this better, and to answer questions in how far the environment plays a role in initiating star formation, better theoretical understanding of the processes involved will be needed (see e.g. Aragón-Calvo et al. (2014).

\section{References}

Aragón-Calvo, M. A. 2007 Morphology and Dynamics of the Cosmic Web, PhD thesis, Univ. Groningen

Aragón-Calvo, M. A, van de Weygaert, R., Araya-Melo, P., Platen, E., \& Szalay, A. S. 2010 $M N R A S$, Vol. 404, p. 89

\&Aragón-Calvo, M. A. and Platen, E., van de Weygaert, R., \& Szalay, A. S. 2010, ApJ, 723, 364

Aragón-Calvo, M. A \&, Szalay, A. S. 2013 MNRAS, 428, p. 3409 
Aragón-Calvo, M. A., Neyrinck M., \& Silk J. 2014, MNRAS, subm, arxiv:1412.1119

\&Beygu, B. and Kreckel, K. and van de Weygaert, R. van der Hulst, J. M., \& van Gorkom, J. H. 2013, AJ, 145, 120

Beygu, B. 2014, The void galaxy survey: a study of the loneliest galaxies in the universe (Ph.D. Thesis, University of Groningen)

Cautun, M., van de Weygaert, R., Jones, B. J.T., \& Frenk, C. S. 2014 MNRAS, Vol. 441, Issue 4, p. 2923

\&Gavazzi, G. and Fumagalli, M. and Galardo, V. and Grossetti, F., Boselli, A., Giovanelli, R., Haynes, M. P., \& Fabello, S. 2012, A\&A, 545, A16

Goldberg, D. M. \& Vogeley, M. 2004 ApJ, 605, p. 1

Hoeft, M. \& Gottlöber, S. AdvAst, 2010, 87

Hoyle, F., Rojas, R. R., Vogeley, M. S., \& Brinkmann, J. 2005, ApJ, 620, 618

Karachentsev, I. D., Makarov, D. I., \& Kaisina, E. I. 2013, AJ, 145, 101

Keres, D., Katz, N., Weinberg, D. H, \& Davé, R. 2005 MNRAS, Vol. 363, p. 2

Kreckel, K., Platen, E., Aragón-Calvo, M. A., van Gorkom, J. H., van de Weygaert, R., van der Hulst, J. M., Kovač, K., Yip, C.-W., \& Peebles, P. J. E. 2011, AJ, 141, 4

Kreckel, K., Platen, E., Aragón-Calvo, M. A., van Gorkom, J. H., van de Weygaert, R., van der Hulst, J. M., \& Beygu, B. 2012, AJ, 144, 16

Lee, J. C., Gil de Paz, A., Tremonti, C., Kennicutt, Jr., R. C., Salim, S., Bothwell, M., Calzetti, D., Dalcanton, J., Dale, D., Engelbracht, C., Funes, S. J. J. G., Johnson, B., Sakai, S., Skillman, E., van Zee, L., Walter, F., \& Weisz, D. 2009, ApJ, 706, 599

Mo, H., van den Bosch, F. C., \& White, S. 2010, Galaxy Formation and Evolution

Peebles, P. J. E. 2001 ApJ, 557, 495

Platen, E., van de Weygaert, R., \& Jones, B. J. T. 2007, MNRAS, 380, 551

Rieder, S., van de Weygaert, R., Cautun, M., Beygu, B., \& Portegies Zwart, S. 2013 MNRAS, 435, p. 222

Sánchez-Gallego, J. R., Knapen, J. H., Wilson, C. D., Barmby, P., Azimlu, M., \& Courteau, S. 2012, MNRAS, 422, 3208

Schaap, W. E. \& van de Weygaert, R. 2000, A\&A, 363, L29

Sheth, R. \& van de Weygaert, R. 2004 MNRAS, 350, p. 517

Stanonik, K., Platen, E., Aragón-Calvo, M. A., van Gorkom, J. H., van de Weygaert, R., van der Hulst, J. M., \& Peebles, P. J. E. 2009, ApJ, 696, L6

van de Weygaert, R., Platen, E. 2011 IJMPS, 1, p. 41

van de Weygaert, R., Kreckel, K., Platen, E., Beygu, B., van Gorkom, J. H, van der Hulst, J. M, Aragón-Calvo, M. A, Peebles, P.J.E., Jarrett, T., Rhee, G., \& Kovac, K. 2011 in Environment and the Formation of Galaxies: 30 years later, ASSP, Springer, p. 17 